\title{
Full-thickness cartilage defects are repaired via a microfracture technique and intraarticular injection of the small-molecule compound kartogenin
}

\author{
Xingquan $\mathrm{Xu}^{1,2}$, Dongquan Shi ${ }^{1,2^{*}}$, Yeshuai Shen ${ }^{1,2}$, Zhihong $X u^{1}$, Jin Dai ${ }^{1,2}$, Dongyang Chen ${ }^{1}$, Huajian Teng ${ }^{2}$ \\ and Qing Jiang ${ }^{1,2^{*}}$
}

\begin{abstract}
Introduction: Microfracture does not properly repair full-thickness cartilage defects. The purpose of this study was to evaluate the effect of intraarticular injection of the small-molecule compound kartogenin (KGN) on the restoration of a full-thickness cartilage defect treated with microfracture in a rabbit model.

Methods: Full-thickness cartilage defects $(3.5 \mathrm{~mm}$ in diameter and $3 \mathrm{~mm}$ in depth) were created in the patellar groove of the right femurs of 24 female New Zealand White rabbits. The rabbits were divided into two groups (12 in each group) based on postsurgery treatment differences, as follows: microfracture plus weekly intraarticular injection of KGN (group 1) and microfracture plus dimethyl sulfoxide (group 2). Six rabbits from each group were illed at 4 and 12 weeks after surgery, and their knees were harvested. The outcome was assessed both macroscopically, by using the International Cartilage Repair Society (ICRS) macroscopic evaluation system, and histologically, by using the modified O'Driscoll histologic scoring system. Immunohistochemistry for type II and I collagen was also conducted.

Results: At 4 weeks, group 1 showed better defect filling and a greater number of chondrocyte-like cells compared with group 2. At 12 weeks, group 1 showed statistically significantly higher ICRS scores and modified O'Driscoll scores compared with group 2. More hyaline cartilage-like tissue was found in the defects of group 1 at 12 weeks.
\end{abstract}

Conclusions: Intraarticular injection of KGN enhances the quality of full-thickness cartilage defects repair after microfracture, with better defect filling and increased hyaline-like cartilage formation.

\section{Introduction}

Full-thickness cartilage defects commonly occur and cannot heal spontaneously because of avascular and hypocellular processes; however, even focal cartilage defects progressively lead to the degeneration of the entire joint. To restore cartilage defects, various kinds of strategies have been developed [1-4]. Microfracture is one of the most common techniques used to treat full-thickness cartilage defects because of its low cost and simple method. After the subchondral bone is perforated to initiate

\footnotetext{
*Correspondence: shidongquan1215@163.com; qingj@nju.edu.cn ${ }^{1}$ The Center of Diagnosis and Treatment for Joint Disease, Drum Tower Hospital, Medical School, Nanjing University, Zhongshan Road 321, Nanjing 210008, Jiangsu, China

${ }^{2} J o i n t$ Research Center for Bone and Joint Disease, Model Animal Research Center (MARC), Nanjing University, Nanjing 210093, Jiangsu, China
}

bleeding, mesenchymal stem cells (MSCs) are recruited into the defects and differentiated into repair tissue. Both research types using rabbit models and short-term clinical studies have demonstrated that microfracture significantly relieves pain and improves knee function [5-7].

However, many researchers have observed that the longterm results after microfracture are poor and that microfracture is limited in treating cartilage defects in elderly patients. Von Keudel et al. [8] conducted a long-term follow-up study to evaluate the results of microfracture by using MRI scans and showed poor outcomes after an average of 48 months after surgery. A meta-analysis conducted by Mithoefer et al. [5] reported that the postoperation revision rate of the microfracture technique was approximately $2.5 \%$ at less than 24 months and increased to $2 \%$ to 
$31 \%$ at 24 months or longer after the operation. Indeed, microfracture does not generate pure hyaline cartilage. Specifically, the main repair tissue after microfracture is fibrocartilage, which primarily contains type I collagen [9-11]. Fibrocartilage is mechanically weaker than hyaline cartilage and degenerates easily $[12,13]$. To generate pure hyaline cartilage without fibrous and hypertrophic tissues in cartilage defects remains challenging.

Numerous studies have been conducted to enhance the hyaline quality of repair tissue in cartilage defects after microfracture surgery. Growth factors, platelet-rich plasma, and various types of scaffolds have been used to improve the repair quality after microfracture [14-17]. Positive effects were observed in the majority of these studies; however, no strategies exist that can fully replicate the biomechanical features of hyaline cartilage because the composition and organization of the repair tissue are insufficient [18]. Furthermore, several reagents and materials that were used in previous studies may even have adverse effects; therefore, novel strategies that can improve the repair quality after microfracture are still needed.

Recently, in a high-throughput screen, Johnson et al. [19] discovered a small molecule, kartogenin (KGN), that directs human bone marrow-derived mesenchymal stem cells (BMSCs) into chondrocytes by mediating the CBF $\beta$-RUNX1 signaling pathway. Two osteoarthritis (OA) models, an acute surgical model, and a collagenase VII-induced model were used in their study to evaluate the repair effect of KGN in vivo. In this study, cartilage matrix regeneration was observed with intraarticular KGN administration. In our previous study, we found that KGN also has the potential to differentiate human synovium-derived mesenchymal stem cells (SMSCs) into chondrocytes. However, no studies have investigated the effect of intraarticular KGN injection on cartilage restoration after microfracture. The purpose of the present study was to evaluate the quality of cartilage-defects repair after microfracture with and without intraarticular KGN injection.

\section{Methods}

In total, 24 skeletally mature female New Zealand White rabbits weighing between 2.0 and $2.5 \mathrm{~kg}$ were used. All the rabbit procedures were approved by the Institutional Rabbit Care and Use Committee of Drum Tower Hospital, Medical School of Nanjing University. KGN was a kind gift from Nanjing Zhenquan Biosciences (Nanjing, China).

\section{Surgical procedure}

Each rabbit was anesthetized with an intramuscular injection of $5 \mathrm{mg}$ droperidol and $0.1 \mathrm{~g}$ ketamine. In addition, anesthetization was maintained by slowly administering ketamine and diazepam through an auricular vein during the operation. The rabbit was placed in the supine position, and a medial parapatellar approach was used to operate on the right knee joint. Then, the patella was dislocated laterally to expose the articular surface. A full-thickness osteochondral defect (3.5 mm in diameter and $3 \mathrm{~mm}$ in depth) was made in the center of the trochlear groove by using an osteoarticular transplantation system. Then microfracture was performed with a $0.35(0.9 \mathrm{~mm})$ Kirschner wire (Figure 1A).

\section{Postoperative treatment}

After surgery, the rabbits were allowed free movement in their cages; their limbs were allowed to bear the entire body weight; and their general health status was monitored by a veterinarian.

\section{KGN and DMSO administration}

The rabbits were randomly divided into the following two groups: the experimental group (Group 1) and the control group (Group 2) (12 in each group). The KGN stock solution was prepared by directly dissolving KGN in DMSO, and the stock solution was diluted $10^{5}$-fold with PBS to a concentration of $10 \mu M$ to prepare the working solution. In total, $0.3 \mathrm{ml}$ of $10 \mu M$ KGN was injected into the operated-on joints of the rabbits in Group 1 once a week for 4 or 12 weeks. The control rabbits received an intraarticular injection of DMSO diluted $10^{5}$-fold. The injection procedure was performed by using a 21-gauge needle and a superior-lateral approach by the same operator.

\section{Macroscopic evaluation}

The rabbits in each group were killed at 4 and 12 weeks (six rabbits at each time interval in each group), and the operated-on knees were harvested. The defect sites were photographed and blindly scored by three different investigators by using the International Cartilage Repair Society (ICRS) scoring system [20] (Table 1).

\section{Histologic evaluation}

After macroscopic evaluation, the specimens were fixed in $10 \%$ formalin for 7 days and decalcified in 15\% ethylenediaminetetraacetate (EDTA)-buffered saline solution (Sunshine, Nanjing, China) for approximately 14 days. The specimens were dehydrated with serial ethanol, embedded in paraffin (Sigma), and cut into $5-\mu \mathrm{m}$ sections. The sections were stained with toluidine blue (Sigma) and safranin $\mathrm{O} /$ Fast Green (Sigma) and viewed under a light microscope (Olympus, ////Japan). The sections were blindly scored by three different investigators by using a modified O'Driscoll histology scoring system [15,21] (Table 2).

\section{Immunohistochemistry}

Regarding the immunohistochemical staining, the sections were incubated with $0.4 \%$ pepsin at $37^{\circ} \mathrm{C}$ for 1 hour for 

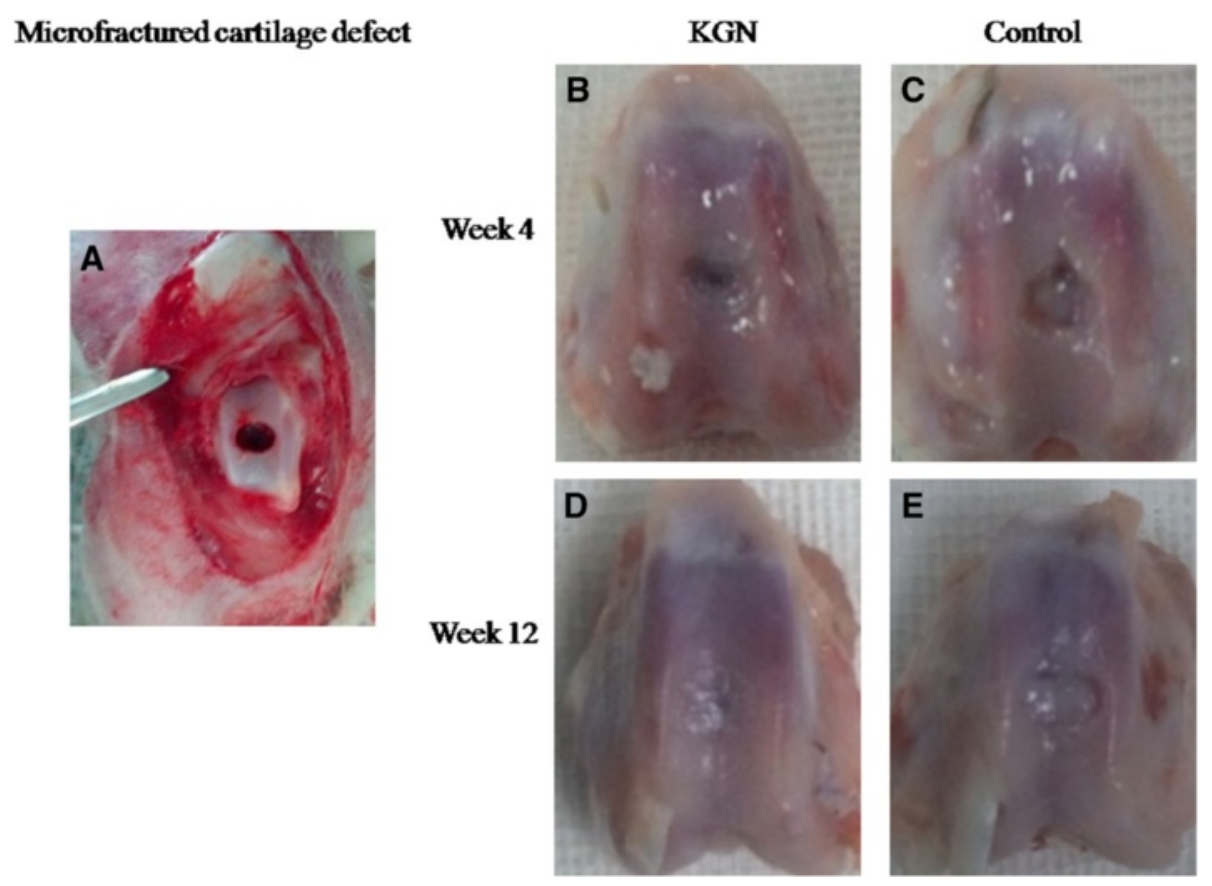

Figure 1 Microfractured cartilage defect (A) and macroscopic appearance of the specimens in groups 1 (B, D) and 2 (C, E). Gross appearance was shown at $4(B, C)$ and 12 weeks (D, E). (B) The repair tissue covered more than 50\% of the cartilage defect. (C) Little repair tissue was observed in the cartilage defect. (D) Repair tissue almost completely covered the defect in the experimental group, but the defect in the control group (E) was insufficiently repaired.

antigen retrieval. Endogenous peroxidase was blocked with $3 \% \mathrm{H}_{2} \mathrm{O}_{2}$ in methanol for 30 minutes. To block nonspecific protein binding, $1 \%$ bovine serum albumin (BSA) solution (Sigma) was used. Monoclonal mouse anti-col II primary antibody (Calbiochem, Merck Millipore, ////1:100 dilution) and anti-col I primary antibody (Abcam) were used to incubate the samples at $4^{\circ} \mathrm{C}$ overnight. Biotinylated secondary anti-mouse antibody (GE Healthcare; 1:500 dilution) was used to incubate the sections for 1 hour. The color reaction was developed with 3',3-diaminobenzidine (DAB) solution (Sigma), and 1\% BSA solution was used as a control.

\section{Data analysis}

All the results are expressed as the mean \pm standard deviation. The differences between the groups for macroscopic and histologic scores were analyzed with the unpaired $t$ test, and a 95\% confidence interval was also calculated. The significance was set at $P \leq 0.05$. All the data were analyzed by using SPSS software (version 20.0; IBM, USA).

\section{Results}

\section{The health status of the rabbits}

No rabbits died because of the surgical procedure or postoperative treatment, and all rabbits were able to load their limbs with no lameness. No evidence of surgery-related complications (e.g., joint or surgery site infection) was observed. No signs of adverse effects related to KGN injection were detected.

\section{Macroscopic evaluation}

At 4 weeks, repair tissue covered more than $50 \%$ of the cartilage defects in group 1; however, relatively little fibrous tissue was observed in group 2 (Figure 1B, C). At 12 weeks, the cartilage defects in group 1 were almost $100 \%$ filled with an obscure demarcation from surrounding cartilage (Figure 1D). However, in group 2, fibrouslike repair tissue covered approximately $80 \%$ of the defects with large penetrating cracks or fissures in many specimens (Figure 1E). At 12 weeks, the mean ICRS score for group 1 was $10.0 \pm 1.1$ compared with a mean score of $7.8 \pm 1.3$ for group $2(\mathrm{P}<0.02)$ (Table 3$)$.

\section{Histological evaluation of the full-thickness cartilage defects treated with microfracture and KGN}

Histological analysis of the repair tissue in group 1 showed the formation of hyaline cartilage-like tissue. At 4 weeks, Toluidine blue staining revealed a larger amount of positively stained repair tissue in the defects in group 1 than in group 2 (Figure 2A, C). At 12 weeks, Toluidine blue staining (Figure 3) and Safranin $\mathrm{O}$ staining (Figure 4A, C and E) were used to assess the proteoglycans content in the repair tissue and showed three grades of intensity. Intense homogeneous staining of Toluidine blue and Safranin O at 12 weeks demonstrated that the defects in the majority 
Table 1 International Cartilage Repair Society macroscopic evaluation of cartilage repair

\begin{tabular}{|c|c|}
\hline Categories & Score \\
\hline \multicolumn{2}{|l|}{ Degree of defect repair } \\
\hline In level with surrounding cartilage & 4 \\
\hline $75 \%$ repair of defect depth & 3 \\
\hline $50 \%$ repair of defect depth & 2 \\
\hline $25 \%$ repair of defect depth & 1 \\
\hline No repair of defect depth & 0 \\
\hline \multicolumn{2}{|l|}{ Integration to border zone } \\
\hline Complete integration with surrounding cartilage & 4 \\
\hline Demarcating border /////_1 mm & 3 \\
\hline $\begin{array}{l}\text { Three-quarters of graft integrated, one-quarter } \\
\text { with a notable border_ } 1 / / / / \mathrm{mm} \text { in width }\end{array}$ & 2 \\
\hline $\begin{array}{l}\text { One-half of graft integrated with surrounding } \\
\text { cartilage, one-half with a notable border///_1 mm }\end{array}$ & 1 \\
\hline $\begin{array}{l}\text { From no contact to one-quarter of graft integrated } \\
\text { with surrounding cartilage }\end{array}$ & 0 \\
\hline \multicolumn{2}{|l|}{ Macroscopic appearance } \\
\hline Intact smooth surface & 4 \\
\hline Fibrillated surface & 3 \\
\hline Small, scattered fissures or cracks & 2 \\
\hline Several small or few large fissures & 1 \\
\hline Total degeneration of grafted area & 0 \\
\hline \multicolumn{2}{|l|}{ Overall repair assessment } \\
\hline Grade I: normal & 12 \\
\hline Grade II: nearly normal & $8-11$ \\
\hline Grade III: abnormal & $4-7$ \\
\hline Grade IV: severely abnormal & $1-3$ \\
\hline
\end{tabular}

of the specimens ( 5 of 6 ) were almost completely reconstructed (Figure 3; Figure 4A, C and E). Both the lateral and basal integration were satisfactory, and the subchondral bone was properly formed. In addition, well organized repair tissue was observed in the best repaired specimen (1 of 6) with nearly complete tidemark formation and nearly normal cell distribution. However, a penetrating crack was still detected in the repair tissue of the least repaired specimen (1 of 6).

\section{Histological evaluation of the full-thickness cartilage defects treated with microfracture and DMSO}

Histological analysis of group 2 showed no hyaline cartilage-like tissue formation. At 4 weeks, Toluidine blue staining (Figure 2B, D) showed that the defects were poorly covered by only fibrous-like tissue. At 12 weeks, both Toluidine blue (Figure 5) and safranin $\mathrm{O}$ (Figure 4B, D and F) slightly and heterogeneously stained the tissue. The demarcation between the repair tissue and the adjacent cartilage remained clear in all the samples. Subchondral bone formation was poor in the majority of the specimens (5 of 6 ). The repair tissue was also poorly organized, and no tidemark formation was observed. At 12 weeks, the histological scores (Table 4) in group 1 were significantly higher than those in group 2 for all the variables except for cartilage degeneration and basal integration, which did not show any significant differences between the two groups. Group 1 showed a significantly higher overall score than group $2(18.5 \pm 4.2$ VS $8.3 \pm 4.6, \mathrm{P}<0.01$ ).

\section{Immunohistochemistry}

In group 1, repair tissue in the majority of the samples showed strong type II collagen staining (Figure 6A) and weak type I collagen staining (Figure $6 \mathrm{C}$ ). In group 2, most of the samples were barely stained with type II collagen (Figure 6B), and intense type I collagen staining was observed (Figure 6D).

\section{Discussion}

This study shows that intra-articular injection of the small molecule KGN after microfracture results in significantly improved repair tissue in cartilage defects. Significant enhancements in the macroscopic ICRS and modified O' Driscoll scores were observed in the experimental group. In addition, the repair tissue that was generated in the presence of KGN showed the characteristics of hyaline cartilage with homogeneous Safranin O staining and strong type II collagen immunoreactivity. Importantly, no adverse effects were caused by the intraarticular KGN injection.

The macroscopic observation of the specimens showed that more repair tissue covered the defects in the experimental group. We speculate that the repair tissue differentiated from the MSCs in bone marrow, synovium or adipose tissue. These MSCs may be recruited into cartilage defects after microfracture [22-24]. Subsequent studies are needed to illuminate the precise roles that each type of MSC plays in repairing these defects. Although BMSCs are no longer enriched when the subchondral bone stops bleeding [25], other sources of MSCs, such as SMSCs in joint fluid, may be recruited during the cartilage repair process [26]. In a previous study, KGN was shown to recruit MSCs in the joint fluid to cartilage damage sites [19]. The intra-articular injection of KGN in our study may enrich MSCs in cartilage defects, which may result in a higher percentage of healing in the experimental group.

In the modified O'Driscoll histology scoring system, the experimental group obtained high scores related to multiple variables, such as the content of hyaline cartilage, the structure of the repair tissue and the reconstruction of the subchondral bone. These data may have resulted from the ability of KGN to promote chondrocyte differentiation from MSCs. Repair tissue and 
Table 2 The modified O'Driscoll histologic score

\section{Characteristic}

I. \% Hyaline cartilage

B. Structural integrity

C. Thickness

D. Bonding to adjacent cartilage

III. Freedom from cellular changes of degeneration

IV. Freedom from degenerate changes in adjacent cartilage

V. Reconstitution of subchondral bone

VI. Bonding of repair cartilage to de novo subchondral bone

VII. Safrinin O staining

$80-100$
$60-80$
$40-60$
$20-40$
$0-20$

Grading

Score

8

6

4

2

0

Smooth and intact

Fissures 2

Severe disruption, fibrillation $\quad 1$

Normal 0

Slight disruption, including cysts 2

Severe lack of integration 1

$100 \%$ of normal adjacent cartilage 0

$50 \%$ to $100 \%$ or thicker than normal 2

0-50\%

Bonded at both ends of graft 0

Bonded at one end/partially both ends 2

Not bonded 1

Normal cellularity, no clusters 0

Slight hypocellularity, $<25 \%$ chondrocyte clusters 2

Moderate hypocellularity, $>25 \%$ clusters 1

Normal cellularity, no clusters, normal staining 0

Normal cellularity, mild clusters, moderate staining 3

Mild or mod hypocellularity, slight staining 2

Severe hypocellularity, slight staining 1

Complete reconstitution 0

Greater than 50\% recon 2

$50 \%$ or less recon 1

Complete and uninterrupted 0

$<100 \%$ but $>50 \%$ recon

$<50 \%$ complete 1

$>80 \%$ homogeneous positive stain 0

40\%-80\% homogeneous positive stain 2

$<40 \%$ homogeneous positive stain 1

Total score 0

Table 3 Results of macroscopic evaluation at 3 months

\begin{tabular}{llllll}
\hline & \multicolumn{2}{l}{ Mean score \pm SD } & & 95\% Confidence interval \\
\cline { 2 - 3 } Time periods & Group 1 & Group 2 & P value & Lower limit & Upper limit \\
\hline Three months & $10.0 \pm 1.1$ & $7.8 \pm 1.3$ & $0.012^{*}$ & 0.60 & 3.73 \\
\hline
\end{tabular}

*Statistically significant deerence. 


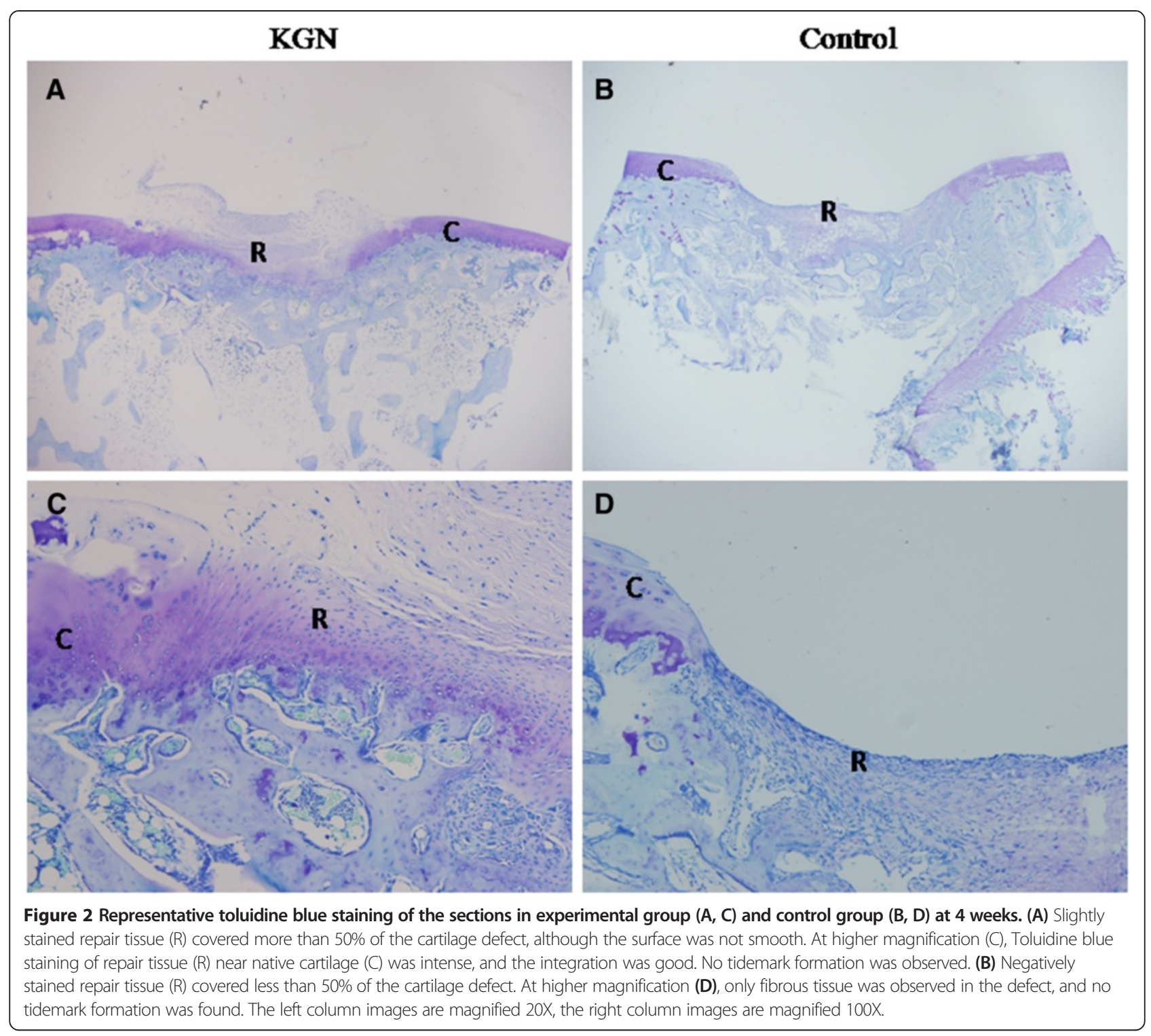

adjacent cartilage regeneration were minimal in group 1 compared with group 2; however, no significant difference was detected. These observations support the data from two previously developed OA models [19]. We postulate that KGN also plays a role in protecting existing chondrocytes. Overall, the efficiency of intraarticular KGN administration after microfracture may be attributable to both repair effect and protective effects.

Intra-articular injection of repair-promoting agents is a simple and less invasive procedure to improve repair tissue after microfracture. Recently, many reagents such as recombinant protein-based growth factors have been used to promote cartilage repair after microfracture [15]. Growth factors have been widely believed to have a strong potency in stimulating chondrogenesis [27,28]. However, small-molecule compounds possess obvious advantages towards recombinant protein-based growth factors. First, the cost and risk of cross-species contamination are significantly lower when using small molecules $[29,30]$. Second, low molecular weight therapeutics is too small $(<1000 \mathrm{KDa})$ to induce an immune response [31]. These properties indicate that small molecule compounds are a potential alternative to growth factors in tissue regeneration $[32,33]$.

The KGN concentration used in our study was $10 \mu \mathrm{M}$ because data from our previous in vitro study showed that $10 \mu \mathrm{M}$ KGN displays the greatest chondrogenesis potential without cytotoxicity. Based on the consideration that KGN sustainably induces MSCs recruited from joint fluid into chondrocytes, the injection procedure was not halted until the rabbits were sacrificed. Therefore, the two duration periods of the injection 


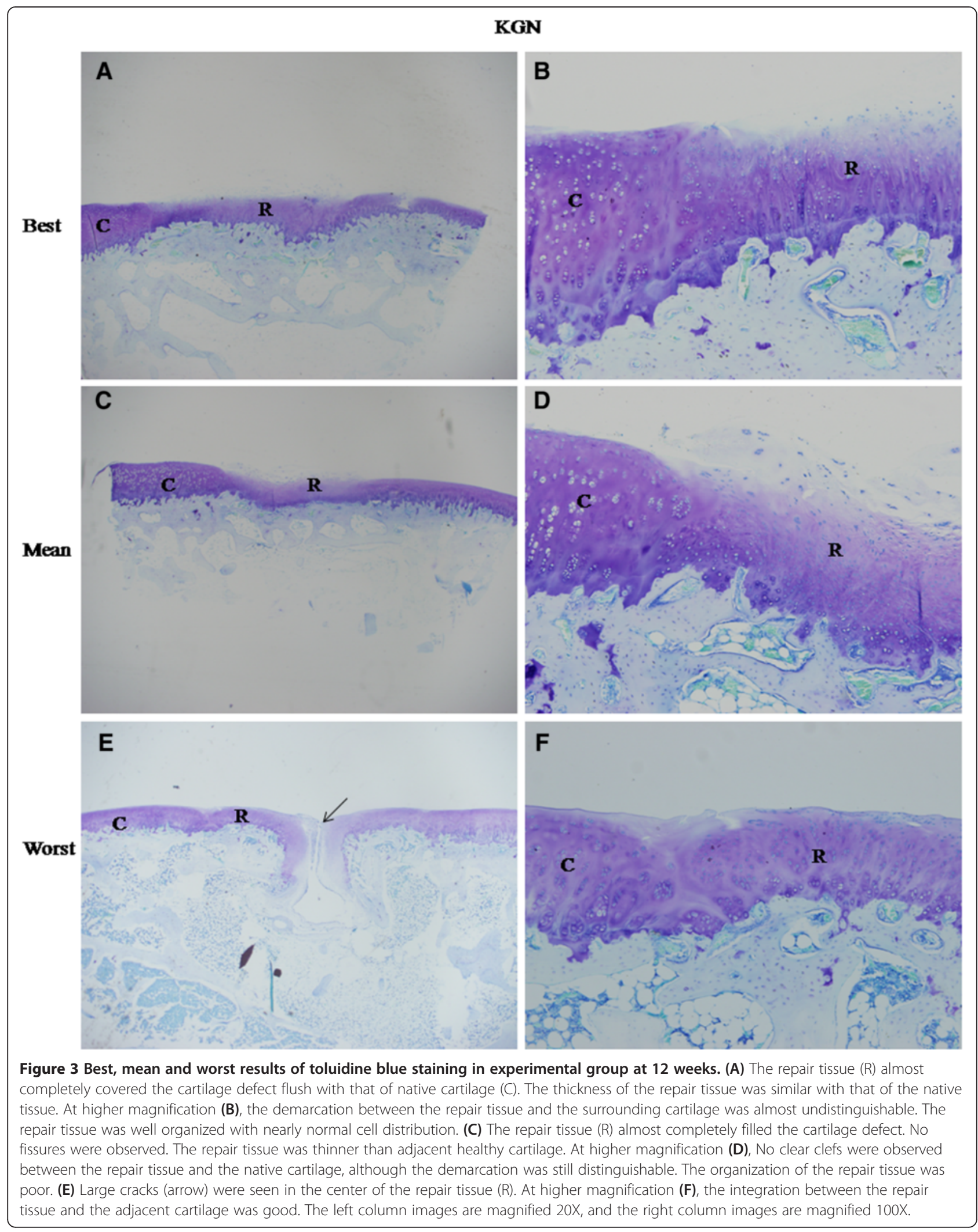




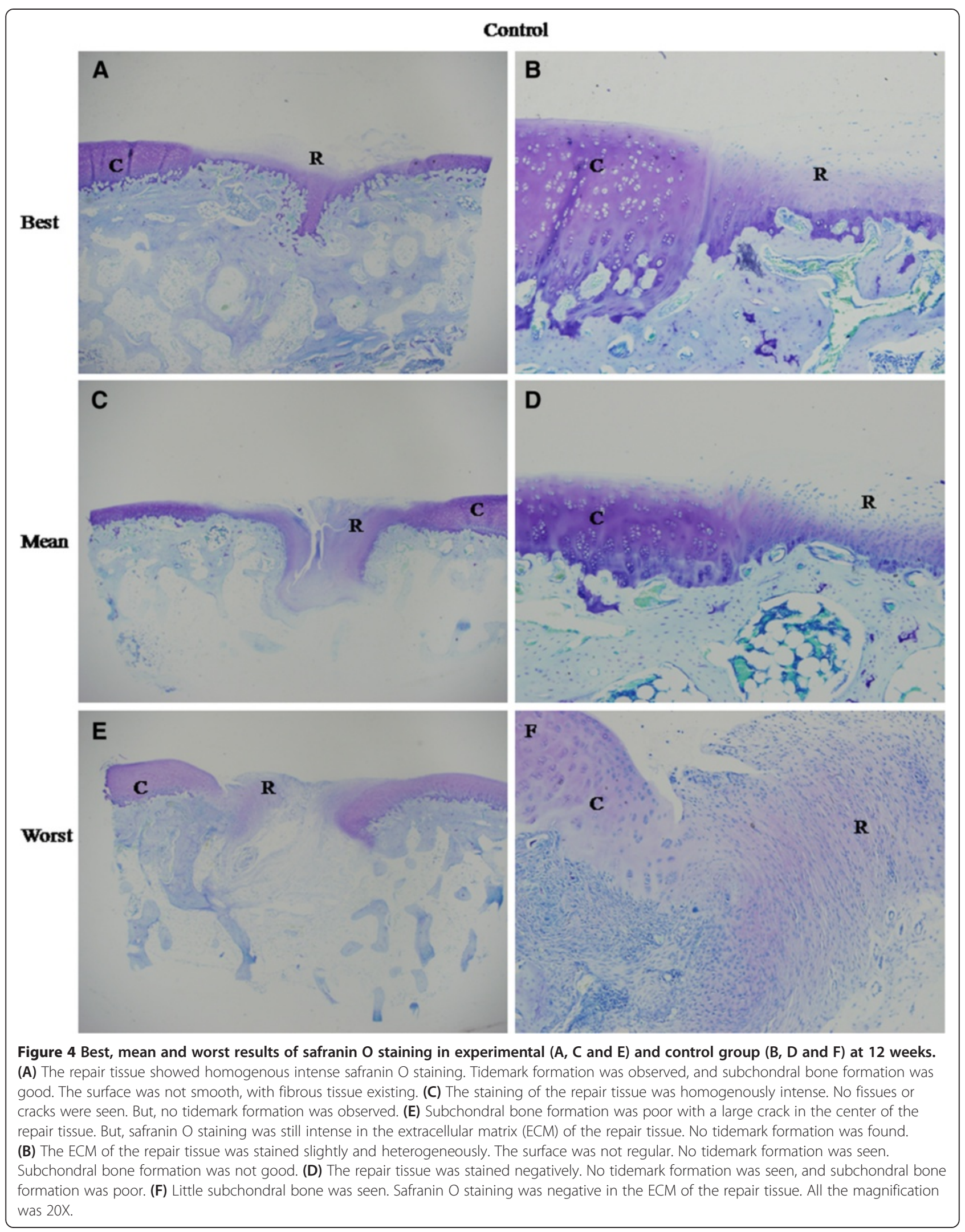




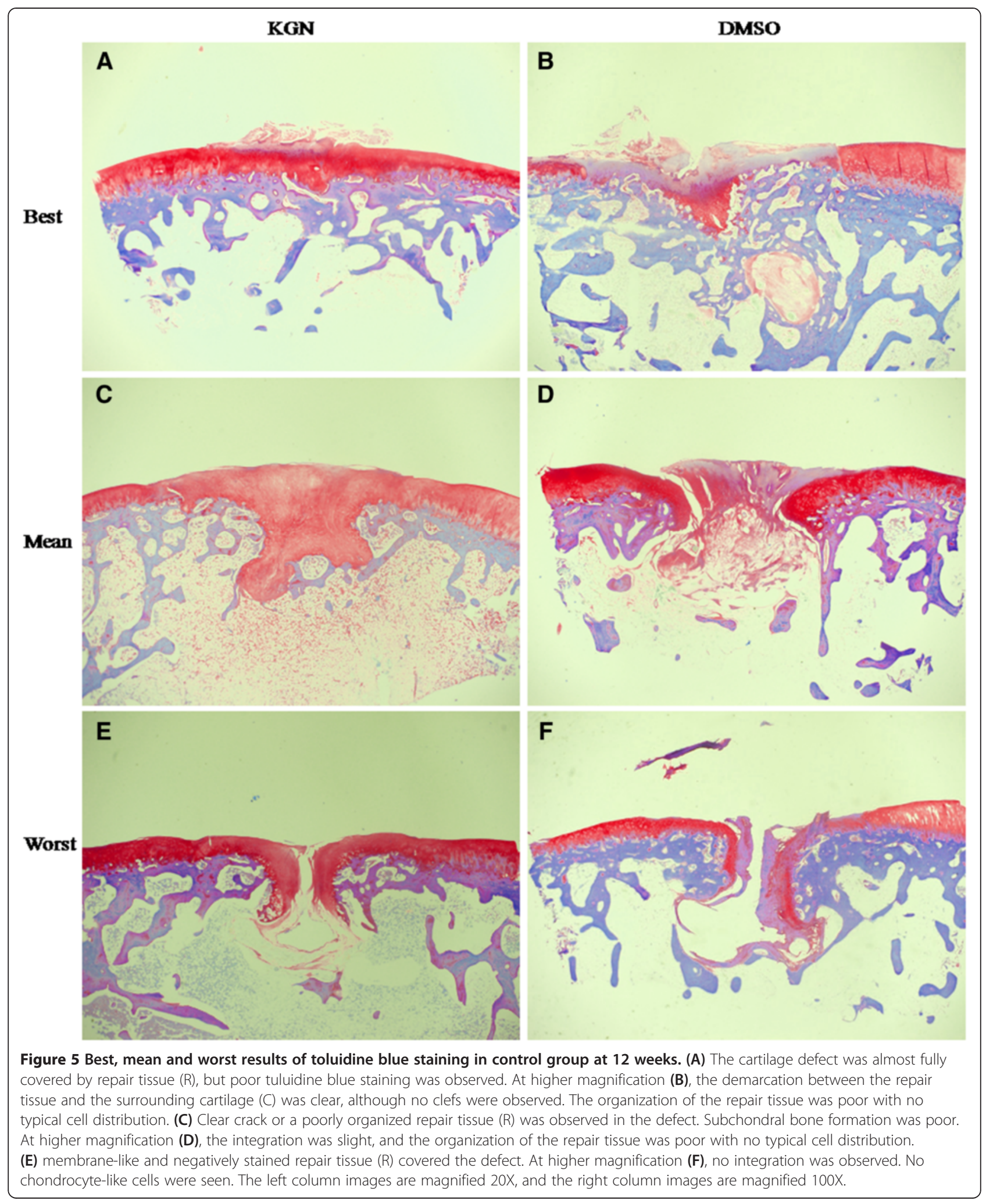


Table 4 Results of histological evaluation at 3 months

\begin{tabular}{|c|c|c|c|c|c|}
\hline \multirow[b]{2}{*}{ Variable } & \multicolumn{2}{|c|}{ Mean score \pm SD } & \multirow[b]{2}{*}{$P$ value } & \multicolumn{2}{|c|}{ 95\% Confidence interval } \\
\hline & Group 1 & $\overline{\text { Group } 2}$ & & Lower limit & Upper limit \\
\hline \%Hyaline cartilage & $4.7 \pm 3.0$ & $1.0 \pm 1.1$ & $0.019^{*}$ & 0.75 & 6.58 \\
\hline Structural characteristics & $6.0 \pm 0.6$ & $3.2 \pm 2.0$ & $0.009^{*}$ & 0.89 & 4.78 \\
\hline Freedom from cellular changes of degeneration & $1.8 \pm 0.4$ & $1.2 \pm 1.0$ & 0.156 & -0.30 & 1.64 \\
\hline Freedom from degenerate changes in adjacent cartilage & $1.7 \pm 0.8$ & $1.3 \pm 0.5$ & 0.419 & -0.55 & 1.21 \\
\hline Reconstitution of subchondral bone & $1.5 \pm 0.6$ & $0.5 \pm 0.6$ & $0.010^{*}$ & 0.30 & 1.71 \\
\hline Bonding of repair cartilage to de novo subchondral bone & $1.5 \pm 0.6$ & $0.8 \pm 0.8$ & 0.110 & -0.18 & 1.53 \\
\hline Safrinin $O$ staining & $1.3 \pm 0.8$ & $0.3 \pm 0.5$ & $0.030^{*}$ & 0.12 & 1.88 \\
\hline Total & $18.5 \pm 4.2$ & $8.3 \pm 4.6$ & $0.003^{*}$ & 4.46 & 15.87 \\
\hline
\end{tabular}

*Statistically significant difference.

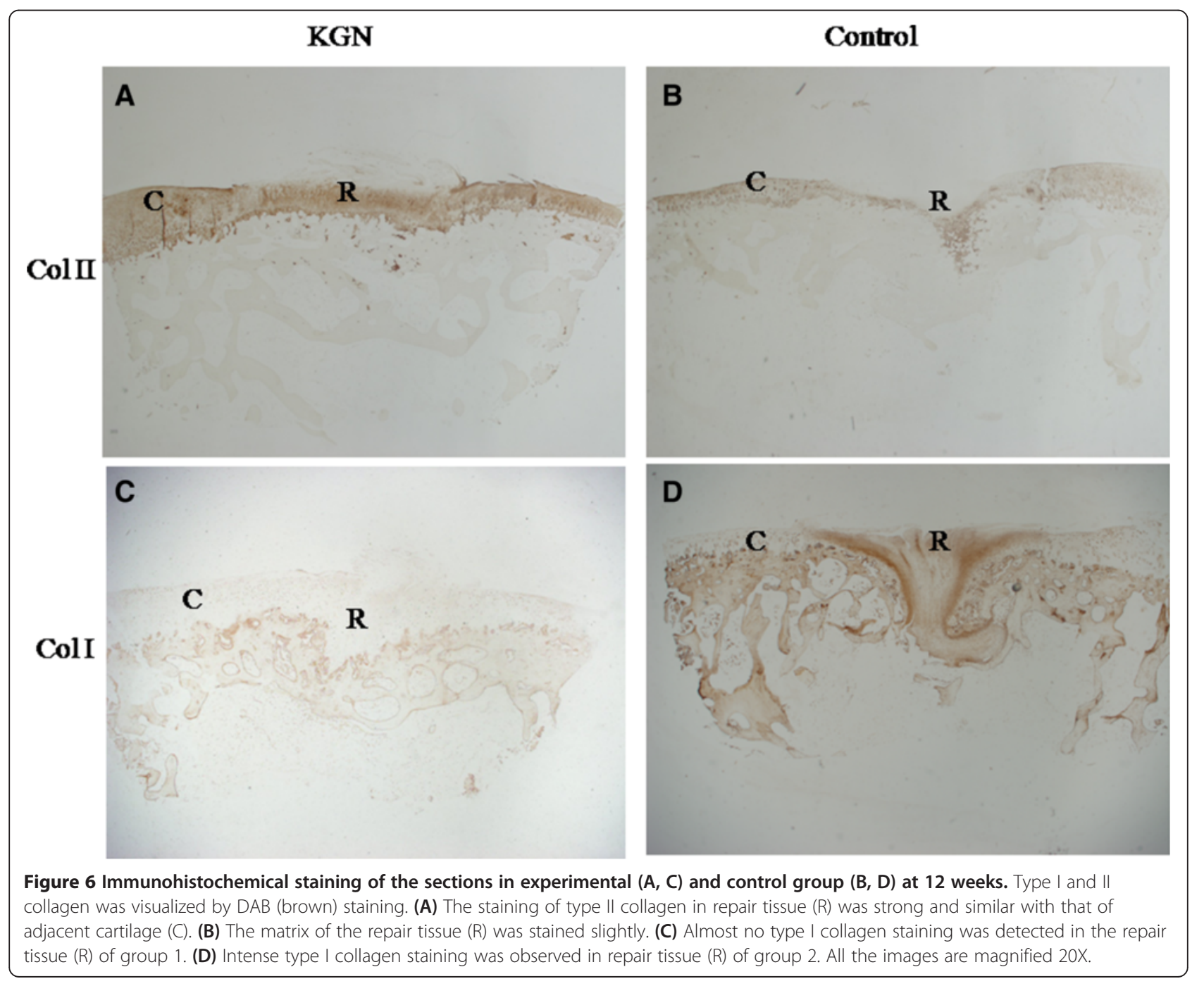


procedure was 4 and 12 weeks. KGN was injected once a week to maintain an effective concentration in the knee joint; however, the precise concentration of KGN in knee joint was not monitored, which is the primary shortcoming of the present study. Future work should focus on identifying the best frequency and duration of KGN injection to promote tissue repair.

\section{Conclusions}

In conclusion, intra-articular KGN injection enhances the quality of full-thickness cartilage defect repair after microfracture.

\section{Abbreviations \\ BMSCs: Bone marrow-derived mesenchymal stem cells; DAB: Diaminobenzidine; ECM: Extracellular matrix; EDTA: Ethylenediaminetetraacetic; KGN: Kartogenin; MSCs: Mesenchymal stem cells; OA: Osteoarthritis; SMSCs: Synovium-derived mesenchymal stem cells.}

\section{Competing interests}

The authors declare that they have no competing interests.

\section{Authors' contributions}

XQX participated in the experimental design, carried out animal surgeries, KGN injections, histological staining, immunohistochemistry staining, analyzed the data, and drafted the manuscript. DQS contributed to conception of the study, experimental design, analyzed the data and drafted the manuscript. YSS helped with the animal surgeries, histological staining and to draft the manuscript. ZHX contributed to the study's conception, analyzed the data and revised the manuscript. JD participated in designing the experiment and revising the manuscript. DYC contributed to conception of the study and revised the manuscript. HJT contributed to conception of the study and helped revise the manuscript. QJ contributed to conception of the study, experimental design, analyzed the data, and revised the manuscript. All authors have read and approved the final manuscript.

\section{Acknowledgement}

This work was supported by the National Nature Science Foundation of China $(81125013,81101338)$ (to D.S. and Q.J.) and the Distinguished Young Investigator Project of Nanjing (to D.S).

Received: 1 August 2014 Accepted: 22 January 2015

Published online: 02 February 2015

\section{References}

1. Chen FS, Frenkel S, Di Cesare P. Repair of articular cartilage defects: part II. Treatment options. Am J Orthop (Belle Mead NJ). 1999;28:88-96.

2. Steadman JR, Rodkey WG, Singleton SB, Briggs KK. Microfracture technique forfull-thickness chondral defects: Technique and clinical results. Oper Tech Orthop. 1997;7:300-4.

3. Krusche-Mandl I, Schmitt B, Zak L, Apprich S, Aldrian S, Juras V, et al. Long-term results 8 years after autologous osteochondral transplantation: 7 T gagCEST and sodium magnetic resonance imaging with morphological and clinical correlation. Osteoarthritis Cartilage. 2012;20:357-63.

4. Moreira-Teixeira LS, Georgi N, Leijten J, Wu L, Karperien M. Cartilage tissue engineering. Endocr Dev. 2011;21:102-15.

5. Mithoefer K, McAdams T, Williams RJ, Kreuz PC, Mandelbaum BR. Clinical efficacy of the microfracture technique for articular cartilage repair in the knee an evidence-based systematic analysis. Am J Sports Med. 2009;37:2053-63.

6. Mithoefer K, WilliamsIII RJ, Warren RF, Potter HG, Spock CR, Jones EC, et al, The microfracture technique for the treatment of articular cartilage lesions in the knee. A prospective cohort study. J Bone Joint Surg. 2005:87:1911-20.

7. Kreuz P, Steinwachs M, Erggelet C, Krause S, Konrad G, Uhl M, et al. Results after microfracture of full-thickness chondral defects in different compartments in the knee. Osteoarthritis Cartilage. 2006;14:1119-25.
8. Von Keudell A, Atzwanger J, Forstner R, Resch H, Hoffelner T, Mayer M. Radiological evaluation of cartilage after microfracture treatment: A long-term follow-up study. Eur J Radiol. 2012;81:1618-24.

9. Frisbie DD, Oxford JT, Southwood L, Trotter GW, Rodkey WG, Steadman JR, et al. Early events in cartilage repair after subchondral bone microfracture. Clin Orthop Relat Res. 2003;407:215-27.

10. Furukawa T, Eyre D, Koide S, Glimcher M. Biochemical studies on repair cartilage resurfacing experimental defects in the rabbit knee. J Bone Joint Surg. 1980;62:79-89.

11. Steadman J, Rodkey W, Briggs K. Microfracture to treat full-thickness chondral defects: surgical technique, rehabilitation, and outcomes. J Knee Surg. 2002;15:170-6.

12. Gilbert JE. Current treatment options for the restoration of articular cartilage. Am J Knee Surg. 1997;11:42-6.

13. Steinwachs M, Guggi T, Kreuz P. Marrow stimulation techniques. Injury. 2008;39:26-31.

14. Milano G, Sanna Passino E, Deriu L, Careddu G, Manunta L, Manunta A, et al. The effect of platelet rich plasma combined with microfractures on the treatment of chondral defects: an experimental study in a sheep model. Osteoarthritis Cartilage. 2010;18:971-80.

15. Power J, Hernandez P, Guehring H, Getgood A, Henson F. Intra-articular injection of rhFGF-18 improves the healing in microfracture treated chondral defects in an ovine model. J Orthop Res. 2014:32:669-76.

16. Doral M, Bilge O, Batmaz G, Donmez G, Turhan E, Demirel M, et al. Treatment of osteochondral lesions of the talus with microfracture technique and postoperative hyaluronan injection. Knee Surg Sports Traumatol Arthrosc. 2012;20:1398-403.

17. Erggelet C, Endres M, Neumann K, Morawietz L, Ringe J, Haberstroh K, et al. Formation of cartilage repair tissue in articular cartilage defects pretreated with microfracture and covered with cell-free polymer-based implants. J Orthop Res. 2009;27:1353-60.

18. Gille J, Schuseil E, Wimmer J, Gellissen J, Schulz A, Behrens P. Mid-term results of autologous matrix-induced chondrogenesis for treatment of focal cartilage defects in the knee. Knee Surg Sports Traumatol Arthrosc. 2010;18:1456-64

19. Johnson K, Zhu S, Tremblay MS, Payette JN, Wang J, Bouchez LC, et al. A stem cell-based approach to cartilage repair. Science. 2012;336:717-21.

20. Van Den Borne M, Raijmakers N, Vanlauwe J, Victor J, de Jong S, Bellemans J, et al. International Cartilage Repair Society (ICRS) and Oswestry macroscopic cartilage evaluation scores validated for use in Autologous Chondrocyte Implantation (ACl) and microfracture. Osteoarthritis Cartilage. 2007:15:1397-402.

21. O'Driscoll SW, Marx RG, Beaton DE, Miura Y, Gallay SH, Fitzsimmons JS. Validation of a simple histological-histochemical cartilage scoring system. Tissue Eng. 2001;7:313-20.

22. Zhang W, Chen J, Tao J, Jiang Y, Hu C, Huang L, et al. The use of type 1 collagen scaffold containing stromal cell-derived factor-1 to create a matrix environment conducive to partial-thickness cartilage defects repair. Biomaterials. 2013:34:713-23.

23. Shapiro F, Koide S, Glimcher M. Cell origin and differentiation in the repair of full-thickness defects of articular cartilage. J Bone Joint Surg. 1993;75:532-53.

24. Erggelet C, Neumann K, Endres M, Haberstroh K, Sittinger M, Kaps C. Regeneration of ovine articular cartilage defects by cell-free polymer-based implants. Biomaterials. 2007;28:5570-80.

25. Caplan Al. The mesengenic process. Clin Plast Surg. 1994;21:429-35.

26. Sekiya I, Ojima M, Suzuki S, Yamaga M, Horie M, Koga H, et al. Human mesenchymal stem cells in synovial fluid increase in the knee with degenerated cartilage and osteoarthritis. J Orthop Res. 2012;30:943-9.

27. Khan IM, Francis L, Theobald PS, Perni S, Young RD, Prokopovich P, et al. In vitro growth factor-induced bio engineering of mature articular cartilage. Biomaterials. 2013;34:1478-87

28. Lee $\mathrm{CH}$, Cook JL, Mendelson A, Moioli EK, Yao H, Mao JJ. Regeneration of the articular surface of the rabbit synovial joint by cell homing: a proof of concept study. Lancet. 2010;376:440-8.

29. Lo KW, Ashe KM, Kan HM, Laurencin CT. The role of small molecules in musculoskeletal regeneration. Regen Med. 2012;7:535-49.

30. Baker MP, Carr FJ. Pre-clinical considerations in the assessment of immunogenicity for protein therapeutics. Curr Drug Saf. 2010;5:308-13.

31. Blaich G, Janssen B, Roth $G$, Salfeld J. Overview: differentiating issues in the development of macromolecules compared with small molecules. In: Handbook of Pharmaceutical Biotechnology. 2007. p. 89-123. 
32. WH Lo K, D Ulery B, Deng M, MAshe K, T Laurencin C. Current patents on osteoinductive molecules for bone tissue engineering. Recent Pat Biomed Eng. 2011;4:153-67.

33. Segar CE, E Ogle M, A Botchwey E. Regulation of angiogenesis and bone regeneration with natural and synthetic small molecules. Curr Pharm Des. 2013;19:3403-19.

Submit your next manuscript to BioMed Central and take full advantage of:

- Convenient online submission

- Thorough peer review

- No space constraints or color figure charges

- Immediate publication on acceptance

- Inclusion in PubMed, CAS, Scopus and Google Scholar

- Research which is freely available for redistribution 